\title{
Forestación de bosques en sabanas de la altillanura colombiana: relevancia de las condiciones ambientales para el establecimiento de plántulas
}

\author{
Afforestation of savanna forests of the Colombian altillanura: relevance of the \\ environmental conditions for the establishment of seedlings
}

\author{
Pablo R. Stevenson, Mónica A. Ramírez, Luisa F. Casas y Francisco Henao-Díaz
}

\section{Resumen}

Una forma de contrarrestar el cambio climático es la generación de bosques con alta capacidad de almacenamiento de carbono. En este trabajo se reportan los resultados de una iniciativa de reforestación de bosque en sabanas de la rserva Tomogrande. Se crearon barreras contra el fuego en seis áreas: dos inundables y dos no inundables, para sembrar plántulas de bosques de la Orinoquia, y manteniendo dos más como control sin adición de plántulas. De 200 plántulas sembradas en sabanas no inundables, únicamente el $17 \%$ de ellas sobrevivieron por más de dos años (33 \% para las especies de bosques aledaños y $12 \%$ para las de otros lugares de la Orinoquia). Ninguna de las 182 plántulas trasplantadas a zonas inundables sobrevivió por más de dos años. La principal causa de mortalidad en sabanas bien drenadas fue el marchitamiento. Se concluye que a pesar de que la ausencia de fuego puede ayudar al establecimiento de árboles, las condiciones climáticas y lumínicas de la sabana disminuyen las probabilidades de que las plantas de bosque se establezcan y las que logran mayor probabilidad de supervivencia son las especies que viven en los bosques de galería aledaños (especialmente en bordes de bosque).

Palabras clave. Barreras de establecimiento. Bosques de galería en Colombia. Sabanas inundables. Supervivencia de plántulas.

\begin{abstract}
One way to counteract climate change is to plant forests with high carbon storage capacity. In this study we report the results of a reforestation project in savannas of the Tomogrande Reserve, in Vichada Department, Colombia. We restricted fire in six one-hectare plots: in two seasonally inundated and two highland savannas we planted seedlings of trees found in the Orinoco Basin and nearby gallery forests and in two of them we did not plant seedlings (control plots). Out of 200 seedlings planted in well-drained savannas, only $17 \%$ survived after two and a half years (33\% came from nearby forest and $12 \%$ from other areas). None of the 182 seedlings planted in flooded savannas survived at the end of the study. The main mortality cause in well-drained savannas was wilting. We concluded that in addition to the control of fire, seedling establishment of forest trees in savannas is highly affected by climatic and light conditions and for this reason the trees with highest potential for restoration are the ones already found at the site in the same ecological conditions (e.g. forest edge).
\end{abstract}

Key words. Flooded savannas. Gallery forests in Colombia. Plant establishment barriers. Plant survival. 


\section{Introducción}

El cambio climático global es un fenómeno bien documentado (Karl y Trenbenth 2003, Meehl et al. 2005) que puede tener fuertes implicaciones en los patrones de diversidad del planeta. Este fenómeno se genera principalmente por el aumento en la concentración de gases de invernadero en la atmósfera y por los efectos de evaporación de la superficie de la tierra (Bonan 2008). Se ha documentado que una de las estrategias para mitigar estos problemas es manteniendo bosques, ya que las plantas por procesos de fotosíntesis pueden fijar $\mathrm{CO}_{2}$ de la atmósfera (Nemani et al. 2000). Sin embargo, es claro que las tendencias actuales en el uso de la tierra son de eliminar bosques para generar zonas productivas (Foley et al. 2005), los cuales tienen una menor capacidad de acumulación de carbono a largo plazo. Ante esta tendencia y otros servicios ambientales que ofrecen los bosques, se ha sugerido que la reforestación y restauración de estos puede ser una alternativa para mitigar los problemas asociados al cambio climático global, que incluyen amenazas a humanos y muchas otras especies (Meehl et al. 2005).

El establecimiento, la supervivencia y crecimiento de las plántulas limitan la regeneración de bosques tropicales (Holl et al. 2000). Por ello es necesario conocer su impacto para evaluar las estrategias más adecuadas para restablecer ambientes degradados o propiciar el establecimiento de árboles en zonas de poca acumulación de biomasa. Entre los factores que influyen en estos procesos están: disponibilidad de semillas, calidad de la dispersión, características edáficas, depredación de semillas, competencia con especies establecidas, disturbios y herbivoría (Holl et al. 2000, Duncan y Chapman 2002).

Uno de los principales factores que afectan el establecimiento de especies vegetales en las sabanas es el régimen de fuegos, el cual ha sido reconocido como una fuerza evolutiva importante para las plantas logrando determinar su distribución, estructura filogenética y procesos de desarrollo (Howe 1995, Silva y Batalha 2010). Las sabanas colombianas por ejemplo, presentan entre el $3 \%$ y el $6 \%$ de los eventos de fuego a nivel mundial (Romero-Ruiz et al 2010) y aunque se considera que tales ecosistemas, han sido sometidos a este tipo de perturbaciones por millones de años, el incremento de fuegos de origen antropogénico ha sido considerable en los últimos tiempos. Lo anterior ha limitado la expansión de los bosques y generado cambios en su estructura y composición; dados los efectos negativos sobre la reproducción y establecimiento de aquellas especies no tolerantes al fuego (Hoffmann et al. 2003).

Se ha propuesto el uso de plantaciones de especies locales como herramienta para atenuar las barreras que limitan la regeneración de los bosques. Entre los beneficios que las plantaciones podrían brindar se encuentran: generar una mayor complejidad estructural y mayor cantidad de recursos. Si se presenta una mayor disponibilidad de recursos, los ecosistemas atraerían mayor cantidad de animales dispersores y aumentaría la lluvia de semillas. Adicionalmente, cuando se establecen bosques mejoran las condiciones microclimáticas del sotobosque que favorecen el reclutamiento de plántulas de árboles de sucesión tardía (Bazzas y Pickett 1980), que requieren distintas condiciones de temperatura, humedad y sombrío. Por otra parte, se mejora la estructura y fertilidad del suelo al crear capas de materia orgánica por la hojarasca de los árboles; se disminuye la competencia de las plántulas bajo el dosel, especialmente con aquellas competidoras como gramíneas y helechos; y se disminuye la probabilidad de disturbios como fuegos (Lugo 1997, Parrota et al. 1997).

Por las razones nombradas anteriormente, las plantaciones de madera han sido una de las estrategias más utilizadas para recuperar áreas degradadas o abandonadas, especialmente por el atractivo económico de las especies maderables. Sin embargo, algunos estudios han probado que bajo ciertas condiciones la regeneración natural puede ser una mejor estrategia para una restauración ecológica, ya que las plantaciones usualmente sólo ayudan a recuperar la capacidad productiva y poco hacen por el restablecimiento de la biodiversidad (Lamb 
1998). Las características que más se debaten acerca del uso de las plantaciones están relacionadas con los efectos negativos de los monocultivos: una baja biodiversidad, una menor estabilidad ecológica, una menor eficiencia en el uso de los recursos, un mayor desgaste de los componentes edáficos e hídricos, y el uso de especies exóticas (Lugo 1997). Es por esto que se ha propuesto el uso de plantaciones como herramientas de forestación sólo bajo ciertas condiciones de alteración de los ecosistemas: zonas muy degradadas, muy extensas, muy alejadas de remanentes de vegetación o dominadas por pastos (Murcia 1997, Lamb 1998).

De cualquier forma, el papel que juegan los cultivos en los procesos de regeneración debe ser evaluado para poder dar apoyo científico a los planes de forestación, tratando de identificar las particularidades propias de cada área geográfica y de cada cultivo usado como estrategia de regeneración. Si se logra un mejor entendimiento de los procesos y los limitantes que gobiernan la regeneración de un bosque intervenido se pueden proponer prácticas que atenúen las barreras, y que agilicen y mejoren la regeneración de los bosques. Teniendo en cuenta el anterior marco conceptual, en este trabajo se quiso realizar un proyecto piloto de forestación de bosques en sabanas de la altillanura en la reserva Tomogrande (Vichada, Orinoquia colombiana). En particular, se centró en evaluar la posibilidad de establecer bosques en sabanas del Orinoco, mediante el control de fuego y forestación asistida mediante la adición de plántulas y plantones.

\section{Material y métodos}

La reserva Tomogrande se encuentra ubicada en el departamento del Vichada en el municipio de Santa Rosalía (451'0,97'N, 70¹6'15,35’W) a 100 m s.n.m. entre los ríos Tomo y caño Grande. La región presenta un régimen climático unimodal, con un intervalo de pluviosidad de anual entre 2.323 y $2.673 \mathrm{~mm}$ (Correa-Gómez y Stevenson 2010), con una época seca de diciembre a marzo y una época de lluvias el resto del año. Sin embargo, para el único año en que se estimó la precipitación anual en el lugar de estudio se obtuvo un valor cercano a los $2.900 \mathrm{~mm}$, correspondiente a un año muy lluvioso.

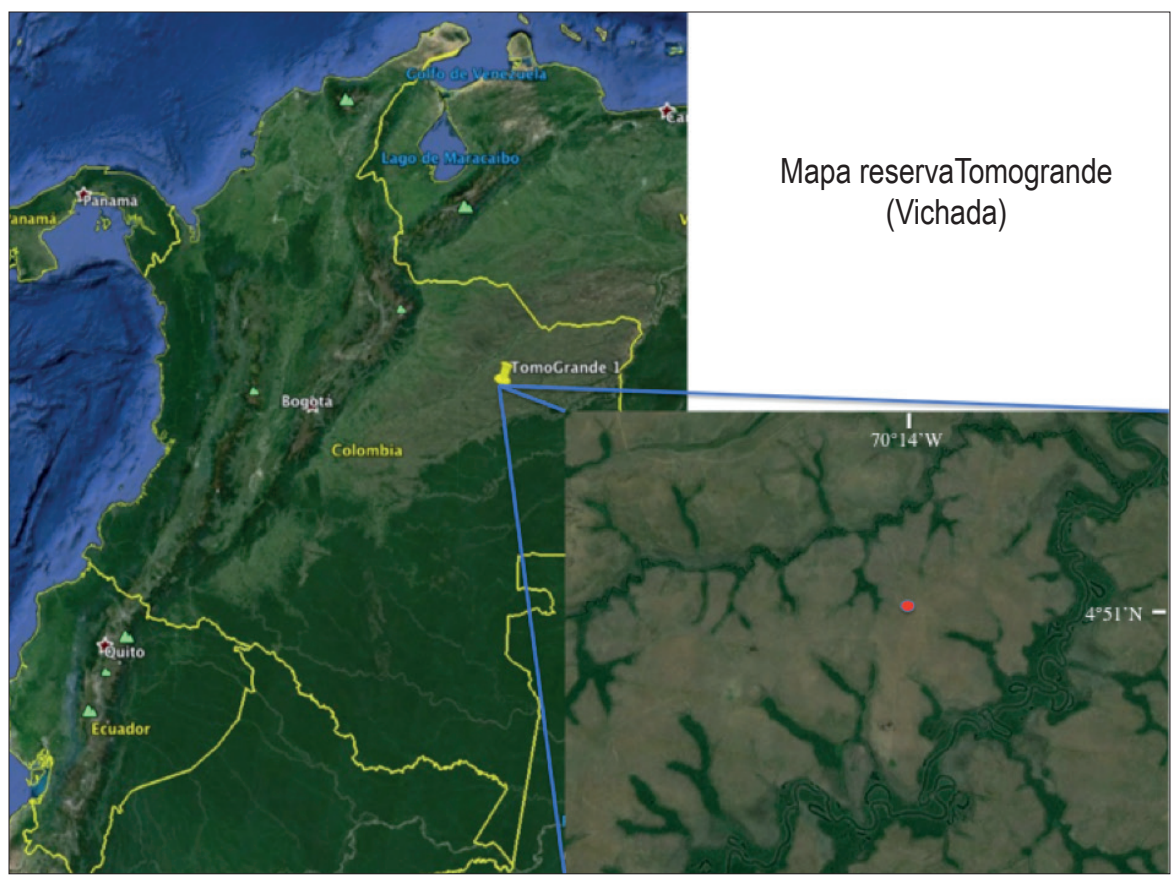

Figura 1. Ubicación geográfica la reserva Tomogrande (Vichada), Orinoquia colombiana. Datos del mapa (2015 Google, Landsat, Copernicus. 
La temperatura promedio anual es de $26{ }^{\circ} \mathrm{C}$, con mínimas de $20{ }^{\circ} \mathrm{C}$ y máximas de $34,5{ }^{\circ} \mathrm{C}$. La topografía presenta leves ondulaciones, con algunas corazas petroférricas de carácter erosivo, y el bioma es considerado en su mayoría peinobioma de sabanas altas (Romero et al. 2004). Así, la zona corresponde a las sabanas de altillanura del Orinoco, que están ubicadas sobre una placa elevada topográficamente que cubre la mayor parte del departamento del Vichada y buena parte del Meta (Castro-Lima et al. 2017), donde ocurren elementos bióticos del escudo Guyanés.

Se instalaron seis parcelas permanentes de vegetación cada una de 1 ha $(100 \times 100 \mathrm{~m})$. Dos de ellas se ubicaron en sabanas de pastos con buen drenaje y cuatro en sabanas arboladas sujetas a inundaciones estacionales (dos sembradas y dos controles). Las parcelas fueron delimitadas con tubos de PVC ubicados cada $10 \mathrm{~m}$ con ayuda de un clinómetro laser con medidor de distancia. Todos los árboles de más de $10 \mathrm{~cm}$ de diámetro a la altura del pecho (DAP) fueron marcados y determinados. También se revisó la presencia de juveniles en 25 subparcelas de $5 \times 5 \mathrm{~m}$, y cobertura de plántulas y herbáceas en 25 subparcelas de $2 \times 2 \mathrm{~m}$. Para clasificar los individuos se siguieron los siguientes criterios: plántulas con altura menor a 1,3 $\mathrm{m}$ ó mayores a $1,3 \mathrm{~m}$ en altura pero con un DAP menor a mayor a $1 \mathrm{~cm}$; juveniles se consideraron plantas con DAP entre 1 y $5 \mathrm{~cm}$ (Stevenson 2011). Las parcelas en sabanas bien drenadas se ubicaron hacia el costado occidental de un bosque de galería a un par de metros de la vegetación leñosa, pues están más protegidas de los vientos y los fuegos. El control del fuego se hizo evitando incendiar las zonas de las parcelas y se instalaron guardarrayas bordeando las zonas a proteger. En estas regiones se cortó la vegetación mediante guadañadora, para mantener una franja de cerca de $5 \mathrm{~m}$ libre de vegetación (Figura 2).

Para la reforestación asistida se compraron 250 plantones de vivero con alturas entre 30 y $60 \mathrm{~cm}$ de altura. El criterio de selección se centró en que fueran especies de la Orinoquia, con énfasis en plantas de frutos carnosos y/o maderables. Sin embargo, también dependió de la disponibilidad en el vivero. De estas se sembraron 200 en julio de 2011 en las parcelas de sabana bien drenada (Tabla 1). Estas plantas fueron sembradas en julio de 2011. En cada esquina se clavó un tubo de PVC y se sembraron tres individuos separados cerca de $50 \mathrm{~cm}$ del tubo. Cada plántula fue marcada con una plaqueta metálica, que fue amarrada con un hilo de nylon para asegurar su monitoreo. Las plántulas se sembraron en las tres líneas más próximas al bosque de galería de la parcela de 100x $100 \mathrm{~m}$, con el fin de reducir el tiempo de exposición al sol directo y a una posible desecación. Se realizó un monitoreo al mes del sembrado y luego una vez al año, revisando la supervivencia y el crecimiento de las plantas hasta el 2013.

Adicionalmente, se utilizaron plántulas del interior de los bosques de galería. Para este efecto se ubicaron plantas creciendo sobre el sistema de trochas de la estación. Estas plántulas fueron removidas cuidadosamente, con el fin de evitar daños en la raíz y fueron trasplantadas inmediatamente a sabanas inundables. Se escogieron este tipo de sabanas, dado que la humedad del suelo podría ayudar a su establecimiento, asumiendo que el requerimiento de agua para estas plantas puede ser muy alto si se encuentran expuestas a sol directo.

\section{Resultados}

Las cuatro parcelas de vegetación de zonas inundables donde se realizó la siembra se caracterizaron por la dominancia del saladillo (Caraipa llanorum), con un máximo de 67 individuos por hectárea (Tabla 2). Esta especie estuvo acompañada de unos pocos individuos esparcidos en la sabana, e incluyendo individuos de chaparro (Curatella americana) y peralejo (Byrsonima crassifolia). La diversidad de las cuatro parcelas fue muy baja, incluyendo apenas entre 3 y 4 especies diferentes por hectárea. En dos de estas parcelas se sembraron plántulas en áreas donde la cobertura de árboles varió entre un 20 y un $50 \%$ (Figura 2). Las parcelas de sabanas bien drenadas no incluyeron ningún individuo de más de $10 \mathrm{~cm}$ de DAP, y tan solo unos pocos individuos esparcidos de chaparro y peralejo. La cobertura de hierbas (principalmente Poaceae) fue mayor al $80 \%$, 
excepto debajo de agrupaciones densas de saladillo. Las plantas más abundantes en las sabanas bien drenadas fueron: Trachypogon spicatus, T. vestitus, Andropogon selloanus, A. leucostachyus y Axonopus purpusii; mientras que las de sabanas inundables fueron: A. anceps, Echinolaena inflexa y Coleataenia caricoides.

De las 200 plántulas sembradas en sabanas no inundables, únicamente el $17 \%$ de las plántulas sobrevivió hasta los dos años y medio (última revisión). Las especies que lograron mayor número de sobrevivientes fueron Simarouba amara, Tapirira guianensis, Caraipa llanorum, Acosmium nitens y Spondias mombin. Las tasas de supervivencia más altas fueron $S$. amara (60\% en dos años y medio), A. nitens y $S$. mombin (50\%) y Caraipa llanorum
(40\%). Todas las especies con números altos de supervivientes están presentes en los bosques aledaños (Correa-Gómez y Stevenson 2012). S. mombin sería una excepción, pero igualmente es común en bosques de várzea de la Orinoquia. Al realizar un análisis de contingencia, mediante una prueba $\mathrm{G}$ de independencia, resulta claro que hay una asociación estadísticamente significativa $(\mathrm{G}=6,52$, $\mathrm{gl} .=1, \mathrm{p}=0,011)$, la cual indica que la probabilidad de sobrevivir es más alta para las especies que crecen en los bosques de la reserva, que para las que no han sido reportadas. Se estima que la tasa de supervivencia de las especies que se encuentran en bosques de galería aledaños fue casi del $33 \%$, y las de otros lugares de la Orinoquia tan solo del $12 \%$ (Tabla 1).
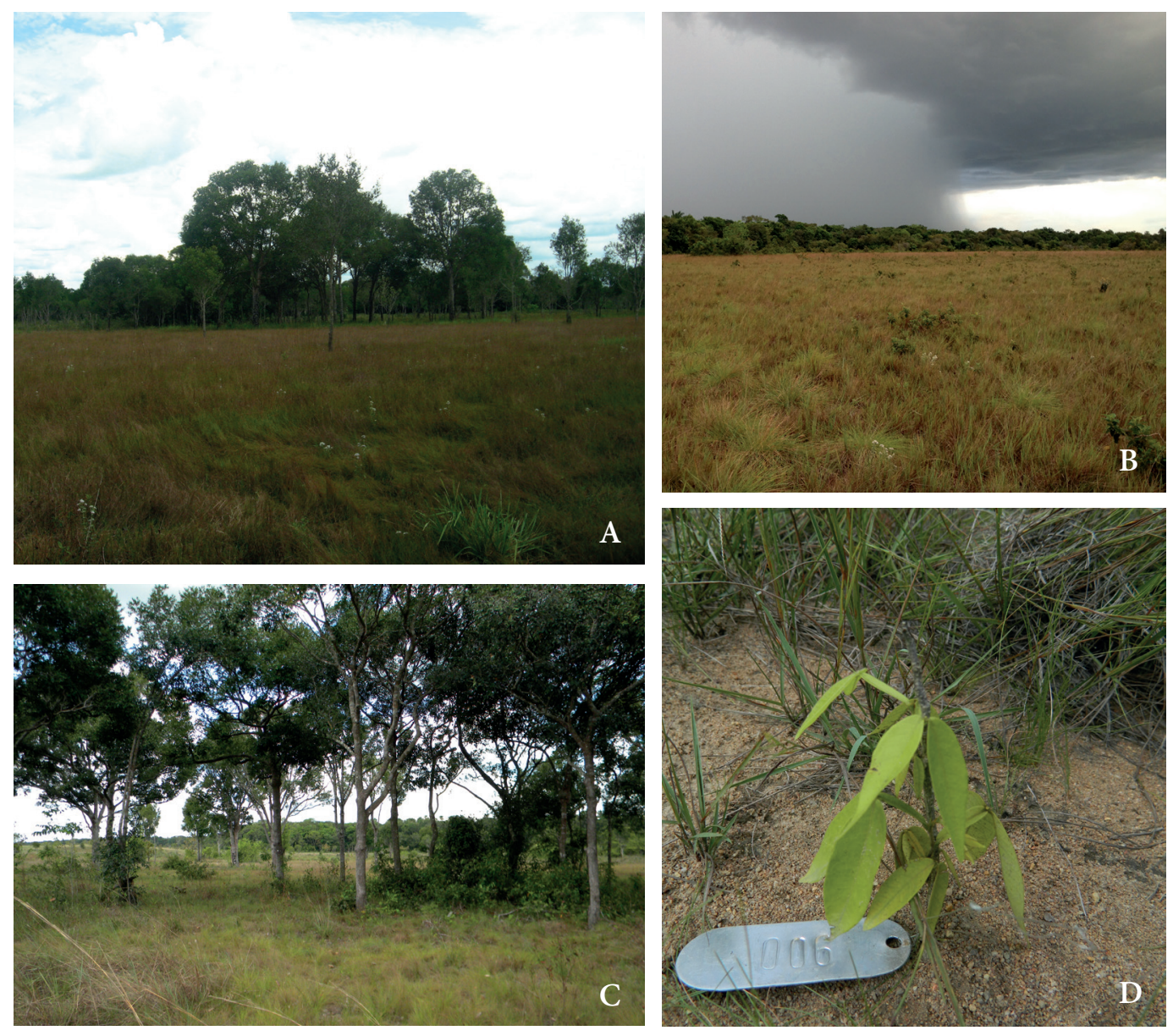

Figura 2. Vista A) sabana inundable, B) sabana no inundable, C) sabana inundable arbolada y D) plántula sembrada. 
Tabla 1. Plantas sembradas en sabanas no inundables en la reserva Tomogrande, Vichada, Colombia. Se incluyen nombres científicos, cantidad de individuos sembrados y sobrevivientes. La última columna indica si la especie ha sido detectada en los boques de la reserva.

\begin{tabular}{|c|c|c|c|}
\hline Especie & $\begin{array}{l}\mathrm{No}^{\circ} \text { plántulas } \\
\text { sembradas } \\
\text { (Jul. 2010) }\end{array}$ & $\begin{array}{c}\text { Número } \\
\text { sobrevivientes } \\
\text { (Dic. 2013) }\end{array}$ & $\begin{array}{l}\text { Presencia en } \\
\text { Tomogrande }\end{array}$ \\
\hline Acosmium nitens & 4 & 2 & $\mathrm{Si}$ \\
\hline Astrocaryum chambira & 13 & 1 & No \\
\hline Attalea butyracea & 15 & 1 & No \\
\hline Campsiandra implexicaulis & 1 & 0 & No \\
\hline Caraipa llanorum & 5 & 2 & $\mathrm{Si}$ \\
\hline Caryodendron orinocense & 2 & 0 & No \\
\hline Chrysophyllum cainito & 3 & 0 & No \\
\hline Enterolobium aff. timbouva & 3 & 1 & No \\
\hline Eschweilera parvifolia & 1 & 0 & $\mathrm{Si}$ \\
\hline Eugenia stipitata & 3 & 0 & No \\
\hline Garcinia madruno & 3 & 1 & $\mathrm{Si}$ \\
\hline Genipa americana & 12 & 0 & $\mathrm{Si}$ \\
\hline Guarea guidonia & 2 & 0 & No \\
\hline Hirtella cf. elongata & 3 & 1 & $\mathrm{Si}$ \\
\hline Inga edulis & 15 & 0 & No \\
\hline Licania pyrifolia & 1 & 0 & No \\
\hline Licania sp. 2 & 1 & 0 & No \\
\hline Mabea nítida & 8 & 0 & $\mathrm{Si}$ \\
\hline Macrolobium acaciifolium & 1 & 0 & No \\
\hline Macrolobium aff. limbatum & 1 & 0 & No \\
\hline Maprounea guianensis & 3 & 1 & $\mathrm{Si}$ \\
\hline Matisia ochrocalyx & 1 & 0 & No \\
\hline Mauritia flexuosa & 19 & 2 & $\mathrm{Si}$ \\
\hline Mouriri guianensis & 1 & 0 & $\mathrm{Si}$ \\
\hline Myrospermum frutescens & 1 & 0 & No \\
\hline Myrtaceae & 1 & 0 & No \\
\hline Ocotea cymbarum & 1 & 0 & No \\
\hline Oenocarpus bacaba & 15 & 2 & $\mathrm{Si}$ \\
\hline Pachira sp. 6 & 6 & 0 & No \\
\hline Perebea xanthochyma & 2 & 0 & No \\
\hline Piranhea trifoliata & 3 & 1 & No \\
\hline Posoqueria aff. latifolia & 5 & 0 & No \\
\hline
\end{tabular}


Cont. Tabla 1. Plantas sembradas en sabanas no inundables en la reserva Tomogrande, Vichada, Colombia. Se incluyen nombres científicos, cantidad de individuos sembrados y sobrevivientes. La última columna indica si la especie ha sido detectada en los boques de la reserva.

\begin{tabular}{lccc}
\hline \multicolumn{1}{c}{ Especie } & $\begin{array}{c}\text { No plántulas } \\
\text { sembradas } \\
\text { (Jul. 2010) }\end{array}$ & $\begin{array}{c}\text { Número } \\
\text { sobrevivientes } \\
\text { (Dic. 2013) }\end{array}$ & $\begin{array}{c}\text { Presencia en } \\
\text { Tomogrande }\end{array}$ \\
\hline Sarcaulus brasiliensis & 1 & 0 & No \\
\hline Simarouba amara & 5 & 3 & $\mathrm{Si}$ \\
\hline Spondias mombin & 4 & 2 & No \\
\hline Stemmadenia grandiflora & 1 & 0 & No \\
\hline Stryphnodendron aff. microstachyum & 10 & 1 & No \\
\hline Swartzia sp. & 3 & 1 & No \\
\hline Tabebuia aff. ochracea & 2 & 0 & No \\
\hline Tachigali sp. & 3 & 1 & $\mathrm{Si}$ \\
\hline Talisia intermedia & 3 & 0 & No \\
\hline Terminalia amazonia & 1 & 0 & No \\
\hline Tetragastris panamensis & 1 & 0 & $\mathrm{Si}$ \\
\hline Indeterminadas & 11 & 11 & \\
\hline
\end{tabular}

Tabla 2. Número de individuos, área basal y DAP de especies arborescentes en parcelas de sabanas inundables.

\begin{tabular}{lcccc}
\cline { 2 - 5 } & \multicolumn{5}{c}{ Parcela } \\
\hline R1 & R2 & R3 & R4 \\
\hline Individuos & 9 & 99 & 9 & 62 \\
\hline Área basal $\left(\mathrm{cm}^{2}\right)$ & 2.103 & 30.562 & 1.906 & 26.596 \\
\hline DAP medio $(\mathrm{cm})$ & 15 & 17 & 15 & 21 \\
\hline Especies & 3 & 3 & 3 & 4 \\
\hline Caraipa llanorum & 7 & 97 & 3 & 59 \\
\hline Curatella americana & 1 & & 3 & \\
\hline Guatteria sp. & & 1 & & \\
\hline Myrcia subsessilis & & 1 & & 1 \\
\hline Tachigali sp. & & & & 1 \\
\hline Licania heteromorpha & & & & \\
\hline Byrsonima crassifolia & 1 & & & \\
\hline
\end{tabular}


El crecimiento de las plántulas sobrevivientes fue muy bajo. De hecho, en promedio la tasa de crecimiento relativo y su variación (altura inicial - altura final / altura inicial), en muchos de los supervivientes fue negativa. Esto ocurrió pues en varios casos las plantas se desecaron, pero lograron producir nuevas yemas en las partes bajas del tronco, decreciendo así en altura las estructuras vivas. En las sabanas bien drenadas, la principal causa de mortalidad fue justamente el marchitamiento.

La supervivencia en zonas inundables fue tan sólo de $14 \%$ durante el primer mes de establecimiento. Las especies que mostraron un mejor establecimiento en la sabana luego del trasplante fueron Goupia glabra, Xylopia aromatica y algunos individuos de Tapirira guianensis. Sin embargo, estas murieron cuando el nivel de las aguas aumentó y las plántulas se vieron sujetas a la inundación. En estas parcelas, muchas de las plántulas botaron sus hojas durante el primer mes de establecimiento, y lograron rebrotar hasta la siguiente creciente de las lluvias. Ninguna de las 182 plántulas sobrevivió por más de dos años, lo que sugiere que ninguna de las especies trasplantadas desde las trochas del bosque está adaptada a las condiciones de inundación (Tabla 3).

\section{Discusión y conclusiones}

Algunos estudios indican que buena parte de las sabanas de la Orinoquia colombiana podrían tener una vegetación arbórea continua, de acuerdo a los patrones de pluviosidad y temperatura de la región y que la presencia de sabanas dependería más de factores antrópicos relacionados con la ocurrencia de fuegos (Henriques y Hoffmann 2011). Si esto fuera cierto, es de esperase que la supresión de fuegos podría ser la principal herramienta para facilitar el establecimiento de especies de árboles que ocurren en la región. En este trabajo, sin embargo, se encontró tanto en los tratamientos de sabanas bien drenadas, como en saladillales inundables, que las tasas de supervivencia fueron muy bajas a pesar de haber realizado un esfuerzo por controlar el efecto del fuego.

Las tasas bajas de establecimiento de plántulas sugiere que las condiciones climáticas son relevantes para tener éxito en planes de generación de bosques en zonas de altillanura; en particular, la altísima mortalidad de plántulas no típicas de la región ( $88 \%$ en dos años), en comparación con la alta mortalidad encontrada para las que sí están reportadas para la zona $(67 \%)$. Lo anterior sugiere que las especies presentes en la región son el resultado de adaptación a condiciones abióticas particulares y no sólo del resultado de procesos azarosos de migración y establecimiento (Hubbell 2001).

De manera similar, la alta mortalidad encontrada en períodos de inundación en las zonas bajas, sugiere que debe tenerse en cuenta especies que posean adaptaciones particulares para soportar este tipo de filtro ecológico (Parolin y Wittmann 2010). La suposición sobre la posibilidad de que especies de interior de bosques de galería podrían verse beneficiadas con la humedad del suelo en sabanas bajas no estuvo soportada por los datos, pues la mortalidad fue del $100 \%$ y del $83 \%$ teniendo en cuenta también sabanas bien drenadas (Tabla 1). Para sabanas inundables, la posibilidad de crecer bajo la sombra de parentales no conllevó a una alta supervivencia, probablemente por las características del drenaje. Sin embargo, no se descarta que para sabanas bien drenadas, la presencia de otras plantas o de especies nodrizas puede ser adecuado para que logren establecerse especies típicas de bosques de galería sobre las sabanas.

Las especies que tuvieron el mayor éxito fueron en su mayoría especies presentes en la zona o especies que crecen bien en condiciones de abundante irradiación, como es el caso de los hobos (Spondias mombin) (Stevenson 2007). Las otras especies con relativo éxito de supervivencia fueron quienes regeneran de manera abundante en bordes de bosque de galería, y por lo tanto, también parecen estar adaptadas a condiciones de alta luminosidad y alto potencial de pérdida de agua en la altillanura colombiana (Stevenson, observaciones personales). Por lo tanto, los resultados aquí obtenidos son consistentes con la idea de que las sabanas de la Orinoquia tienen una larga historia (Behling y Hooghiemstra 1999), durante la cual las poblaciones de árboles han desarrollado adaptaciones a ambientes particulares. 
Tabla 3. Especies utilizadas en la forestación de sabanas inundables. Las siglas corresponden a morfoespecies.

\begin{tabular}{|c|c|c|}
\hline Especie & Familia & Número \\
\hline Bocageopsis multiflora & Annonaceae & 1 \\
\hline Clarisia racemosa & Moraceae & 2 \\
\hline Cupania cf. scrobiculata & Sapindaceae & 1 \\
\hline Goupia glabra & Goupiaceae & 8 \\
\hline Guatteria cf. foliosa & Annonaceae & 11 \\
\hline Inga cf. alba & Fabaceae & 7 \\
\hline Inga sp. MR & Fabaceae & 3 \\
\hline Inga sp. Peq & Fabaceae & 1 \\
\hline Inga sp. RA & Fabaceae & 7 \\
\hline Inga sp. SD & Fabaceae & 2 \\
\hline Irianthera laevis & Myristicaceae & 5 \\
\hline Lauraceae & Lauraceae & 1 \\
\hline Laurel alargado & Lauraceae & 1 \\
\hline Licania subarachnophylla & Chrysobalanaceae & 1 \\
\hline Maprounea guianensis & Euphorbiaceae & 1 \\
\hline Melastomataceae & Melastomataceae & 3 \\
\hline Miconia cf. punctata & Melastomataceae & 1 \\
\hline Ocotea sp. & Lauraceae & 1 \\
\hline Oenocarpus bataua & Arecaceae & 12 \\
\hline Pourouma aurea & Urticaceae & 3 \\
\hline Pourouma bicolor & Urticaceae & 1 \\
\hline Pseudolmedia laevis & Moraceae & 2 \\
\hline Simarouba amara & Simaroubaceae & 7 \\
\hline Tapirira guianensis & Anacardiaceae & 28 \\
\hline Tetragastris sp. & Burseraceae & 2 \\
\hline Virola cf. elongata & Myristicaceae & 5 \\
\hline Virola sebifera & Myristicaceae & 1 \\
\hline Vismia cf. minutiflora & Hypericaceae & 1 \\
\hline Xylopia aromatica & Annonaceae & 23 \\
\hline Indeterminados & & 38 \\
\hline
\end{tabular}


Dado que la causa de muerte más común para las plántulas sembradas en la sabana bien drenada fue el marchitamiento, se recomienda que en futuros trabajos de forestación o restauración se debe procurar que las plántulas puedan superar la barrera de la desecación. Las sabanas bien drenadas no tienen períodos de más de un día de inundación (se pueden inundar parcialmente durante una gran lluvia), pero en épocas secas se genera un alto estrés hídrico para las plantas, que pareció ser la principal causa de mortalidad. A pesar de que otros autores han resaltado que la competencia con pastos (Hanan y Lehmann 2010) y la escasez de nutrientes en este tipo de sabanas (Medina y Silva 1990) son factores importantes en el establecimiento de plántulas de árboles, se considera que la aplicación de plantas nodriza y especialmente el suministro de agua son claves para el éxito de establecimiento en sabanas.

De este proyecto piloto, las principales conclusiones indican que los planes de forestación de las sabanas del Orinoco deben tener en cuenta especies adaptadas a ambientes particulares. Aunque se considera que el proyecto fue poco exitoso, esperamos que la experiencia pueda aportar para futuros trabajos de forestación en este tipo de ecosistemas, dado que es muy poco lo que se ha publicado al respecto (Mendez et al. 2012). A partir de esta experiencia, se sugiere que la principal barrera para el establecimiento (una vez controlado el fuego), parece ser la posibilidad de marchitamiento por falta de agua o el exceso de ella en sabanas inundables. Se cree que futuros trabajos deben incluir suministro de agua en períodos de escasez o disminución de la radiación por medio de especies nodriza, propias de la región.

\section{Agradecimientos}

Queremos agradecer a Nelson Galvis, María Fernanda Torres, Sofía Argüello y David Góngora por su colaboración en el trabajo de campo. También a John y Luz Dary, por la colaboración manteniendo los guarda-rayas. A Francisco Castro por el aporte del material vegetal, por sus determinaciones y por los comentarios sobre el documento final. Este estudio fue financiado por un proyecto semilla de la Facultad de Ciencias de la Universidad de Los Andes.

\section{Bibliografía}

Bazzaz, F. A. y S. T. A. Pickett. 1980. Physiological ecology of tropical succession: a comparative review. Annual review of ecology and systematics 11: 287-310.

Behling, H. y H. Hooghiemstra. 1999. Environmental history of the Colombian savannas of the Llanos Orientales since the Last Glacial Maximum from lake records El Pinal and Carimagua. Journal of Paleolimnology 21: 461-476.

Bonan, G. B. 2008. Forests and climate change: Forcings, feedbacks, and the climate benefits of forests. Science 320 (5882): 1444-1449.

Castro-Lima, F., R. Antelo-Albertos, P. R. Stevenson y M. González-Caballero. 2016. Ecosistémas estratégicos. Pp: 33-56. En: Trujillo, F., R. Antelo y S. Usma (Ed). Biodiversidad de la cuenca baja y media del río Meta. Fundación Omacha, Fundación Palmarito y WWF. Bogotá.

Correa-Gomez, D. F. y P. R. Stevenson. 2010. Estructura y diversidad de bosques de galería de una sabana estacional de los llanos orientales colombianos (Reserva Tomo Grande, Vichada). Revista Orinoquia 14 (1): 31-48.

Duncan, S. y C. Chapman. 2002. Limitations of animal seed dispersal for enhancing forest succession on degraded lands. Pp: 437-450. En: Levey, D., W. Silva y M. Galetti. (Ed.). Seed Dispersal and Frugivory: Ecology, Evolution and Conservation. CAB International, Wallingford, UK.

Foley, J. A., R. Defries, G. P. Asner, C. Barford, G. Bonan, S. R. Carpenter, F. S. Chapin, M. T. Coe, G. C. Daily, H. K. Gibbs, J. H. Helkowski, T. Holloway, E. A. Howard, C. J. Kucharik, C. Monfreda, J. A. Patz, I. C. Prentice, N. Ramankutty y P. Snyder. 2005. Global consequences of land use. Science 309 (5734): 570-574.

Hanan, N. y C. Lehmann. 2010. Tree-Grass Interactions in Savannas: Paradigms, Contradictions, and Conceptual Models. Taylor and Francis Group: Boca Raton, FL, USA. 56 pp.

Henriques, R. P. B. y W. A. Hoffmann. 2011. Tree increase in fire-protected cerrado savanna: dynamics and implications to disequilibrium hypothesis. Abstract. ATBC \& SCB Africa, meeting program Arusha, Tanzania.74 pp.

Holl, K., M. Loik, E. Lin e I. Samuels. 2000. Tropical Montane Forest Restoration in Costa Rica: Overcoming Barriers to Dispersal and Establishment. Restoration Ecology 8 (4): 339-349.

Howe, H. F. 1995. Succession and fire season in experimental prairie plantings. Ecology 76 (6): 1917-1925.

Karl, T. R. y K. E. Trenberth. 2003. Modern global climate change. Science 302 (5651): 1719-1723.

Lamb, D. 1998. Large-scale ecological restoration of degraded tropical forest lands: The potential role of timber plantations. Restoration Ecology 6 (3): 271-279. 
Lugo, A. 1997. The Apparent Paradox or Reestablishing Species Richness on Degraded Lands on Tree Monocultures. Forest Ecology and Management 99: 9-19.

Medina, E. y J. F. Silva. 1990. Savannas of northern South America: a steady state regulated by water-fire interactions on a background of low nutrient availability. Journal of Biogeography 17: 403-413.

Méndez, F. F., Y. K. C. Martínez y M. B.Sarmiento. 2012. Biodiversidad vegetal asociada a plantaciones forestales de Pinus caribaea Morelet y Eucalyptus pellita F. Muell establecidas en Villanueva, Casanare, Colombia. Revista Facultad Nacional de Agronomía, Medellín 65 (2): 6749-6764.

Meehl, G. A., W. M. Washington, W. D. Collins, J. M. Arblaster, A. X. Hu, L. E. Buja, W. G. Strand y H. Y. Teng. 2005 . How much more global warming and sea level rise? Science 307 (5716): 1769-1772.

Murcia, C. 1997. Evaluation of Andean Alder as a catalyst for the recovery of tropical cloud forests in Colombia. Forest Ecology and Management 99: 163-170.

Nemani, R. R., C. D. Keeling, H. Hashimoto, W. M. Jolly, S. C. Piper, C. J. Tucker, R. B. Myneni y S. W. Running. 2003. Climate-driven increases in global terrestrial net primary production from 1982 to 1999. Science 300 (5625): 1560-1563.

Pablo R. Stevenson

Universidad de Los Andes,

Facultad de Ciencias,

Laboratorio de Ecología de Bosques Tropicales y Primatología,

Centro de Investigaciones Ecológicas La Macarena

Bogotá, Colombia

pstevens@uniandes.edu.co

Mónica A. Ramírez

Universidad de Los Andes,

Facultad de Ciencias,

Laboratorio de Ecología de Bosques Tropicales y Primatología,

Centro de Investigaciones Ecológicas La Macarena

Bogotá, Colombia

ma.ramirez1101@uniandes.edu.co

Luisa F. Casas

Universidad de Los Andes,

Facultad de Ciencias,

Laboratorio de Ecología de Bosques Tropicales y Primatología,

Centro de Investigaciones Ecológicas La Macarena

Bogotá, Colombia

lfcasascaro@gmail.com

Francisco Henao-Díaz

Universidad de Los Andes,

Facultad de Ciencias,

Laboratorio de Ecología de Bosques Tropicales y Primatología,

Centro de Investigaciones Ecológicas La Macarena

Bogotá, Colombia

lf.henao28@uniandes.edu.co
Parolin, P. y F. Wittmann. 2010. Struggle in the flood: tree responses to flooding stress in four tropical floodplain systems. AoB plants: plq003. doi: 10.1093/aobpla/plq003.

Parrota, J., J. Turnbull y N. Jones. 1997. Catalyzing native forest regeneration on degraded tropical lands. Forest Ecology and Management 99: 1-7.

Romero, M., G. Galindo, J. Otero y D. Armenteras. 2004. Ecosistemas de la cuenca del Orinoco colombiano. Instituto de Investigación de Recursos Biológicos Alexander von Humboldt. Bogotá. Colombia. 189 pp.

Romero-Ruiz, M., A. Etter, A. Sarmiento y K. Tansey. 2010. Spatial and temporal variability of fires in relation to ecosystems, land tenure and rainfall in savannas of northern South America. Global Change Biology 16: 2013-2023. doi: 10.1111/j.1365-2486.2009.02081.x

Silva, I. A. y M. A. Batalha. 2010. Phylogenetic structure of Brazilian savannas under different fire regimes. Journal of Vegetation Science 21 (6): 1003-1013.

Stevenson, P. R. 2007. A test of the escape and colonization hypotheses for zoochorous tree species in a Western Amazonian forest. Plant Ecology 190 (2): 245-258.

Sevenson, P. R. 2011. The abundance of large ateline monkeys is positively associated with the diversity of plants regenerating in Neotropical Forests. Biotropica 43 (4): 512-519.

Forestación de bosques en sabanas de la altillanura colombiana: relevancia de las condiciones ambientales para el establecimiento de plántulas

Citación del artículo: Stevenson, P. R., M. A. Ramírez, L. F. Casas y F. Henao-Díaz. 2017. Forestación de bosques en sabanas de la altillanura colombiana: relevancia de las condiciones ambientales para el establecimiento de plántulas. Biota Colombiana 18 (Suplemento 1): 99-109. DOI: 10.21068/ c2017.v18s01a06

Recibido: 23 de agosto de 2014

Aprobado: 31 de enero de 2017 


\title{
Guía para autores
}

\author{
(humboldt.org.co/es/bibliotecaypublicaciones/biota)
}

\section{Preparación del manuscrito}

El envío de un manuscrito implica la declaración explícita por parte del autor(es) de que este no ha sido previamente publicado, ni aceptado para su publicación en otra revista $u$ otro órgano de difusión científica. Todas las contribuciones son de la entera responsabilidad de sus autores y no del Instituto de Investigación de Recursos Biológicos Alexander von Humboldt, ni de la revista o sus editores.

Los trabajos pueden estar escritos en español, inglés o portugués, y se recomienda que no excedan las 40 páginas (párrafo espaciado a 1,5 líneas) incluyendo tablas, figuras y anexos. En casos especiales el editor podrá considerar la publicación de trabajos más extensos, monografías o actas de congresos, talleres o simposios. De particular interés para la revista son las descripciones de especies nuevas para la ciencia, nuevos registros geográficos y listados de la biodiversidad regional.

Para la elaboración de los textos del manuscrito se puede usar cualquier procesador de palabras (preferiblemente Word); los listados (a manera de tabla) deben ser elaborados en una hoja de cálculo (preferiblemente Excel). Para someter un manuscrito es necesario además anexar una carta de intención en la que se indique claramente:

1. Nombre completo del (los) autor (es), y direcciones para envío de correspondencia (es indispensable suministrar una dirección de correo electrónico para comunicación directa).

2. Título completo del manuscrito.

3. Nombres, tamaños y tipos de archivos suministrados.

4. Lista mínimo de tres revisores sugeridos que puedan evaluar el manuscrito, con sus respectivas direcciones electrónicas.

\section{Evaluación del manuscrito}

Los manuscritos sometidos serán revisados por pares científicos calificados, cuya respuesta final de evaluación puede ser: a) aceptado (en cuyo caso se asume que no existe ningún cambio, omisión o adición al artículo, y que se recomienda su publicación en la forma actualmente presentada); b) aceptación condicional (se acepta y recomienda el artículo para su publicación solo si se realizan los cambios indicados por el evaluador); y c) rechazo (cuando el evaluador considera que los contenidos o forma de presentación del artículo no se ajustan a los requerimientos y estándares de calidad de Biota Colombiana).

\section{Texto}

- Para la presentación del manuscrito configure las páginas de la siguiente manera: hoja tamaño carta, márgenes de $2,5 \mathrm{~cm}$ en todos los lados, interlineado 1,5 y alineación hacia la izquierda (incluyendo título y bibliografía).

- Todas las páginas de texto (a excepción de la primera correspondiente al título), deben numerarse en la parte inferior derecha de la hoja.
- Use letra Times New Roman o Arial, tamaño 12 puntos en todos los textos. Máximo 40 páginas, incluyendo tablas, figuras y anexos. Para tablas cambie el tamaño de la fuente a 10 puntos. Evite el uso de negritas o subrayados.

- Los manuscritos debe llevar el siguiente orden: título, resumen y palabras clave, abstract y key words, introducción, material y métodos, resultados, discusión, conclusiones (optativo), agradecimientos (optativo) y bibliografía. Seguidamente, presente una página con la lista de tablas, figuras y anexos. Finalmente, incluya las tablas, figuras y anexos en archivos separadas, debidamente identificadas.

- Escriba los nombres científicos de géneros, especies y subespecies en cursiva (itálica). Proceda de la misma forma con los términos en latín (p. e. sensu, et al.). No subraye ninguna otra palabra o título. No utilice notas al pie de página.

- En cuanto a las abreviaturas y sistema métrico decimal, utilice las normas del Sistema Internacional de Unidades (SI) recordando que siempre se debe dejar un espacio libre entre el valor numérico y la unidad de medida (p. e. $16 \mathrm{~km}, 23^{\circ} \mathrm{C}$ ). Para medidas relativas como m/seg., use m.seg-1.

- Escriba los números del uno al diez siempre con letras, excepto cuando preceden a una unidad de medida (p. e. $9 \mathrm{~cm}$ ) o si se utilizan como marcadores (p. e. parcela 2, muestra 7).

- No utilice punto para separar los millares, millones, etc. Utilice la coma para separar en la cifra la parte entera de la decimal ( $\mathrm{p}$. e. 3,1416). Enumere las horas del día de 0:00 a 24:00.

- Exprese los años con todas las cifras sin demarcadores de miles (p. e. 1996-1998). En español los nombres de los meses y días (enero, julio, sábado, lunes) siempre se escriben con la primera letra minúscula, no así en inglés.

- Los puntos cardinales (norte, sur, este y oeste) siempre deben ser escritos en minúscula, a excepción de sus abreviaturas $\mathrm{N}, \mathrm{S}$, $\mathrm{E}, \mathrm{O}$ (en inglés $\mathrm{W}$ ), etc. La indicación correcta de coordenadas geográficas es como sigue: $02^{\circ} 37^{\prime} 53^{\prime \prime} \mathrm{N}-56^{\circ} 28^{\prime} 53^{\prime \prime} \mathrm{O}$. La altitud geográfica se citará como se expresa a continuación: $1180 \mathrm{~m}$ s.n.m. (en inglés $1180 \mathrm{~m}$ a.s.l).

- Las abreviaturas se explican únicamente la primera vez que son usadas.

- Al citar las referencias en el texto mencione los apellidos de los autores en caso de que sean uno o dos, y el apellido del primero seguido por et al. cuando sean tres o más. Si menciona varias referencias, éstas deben ser ordenadas cronológicamente y separadas por comas (p. e. Rojas 1978, Bailey et al. 1983, Sephton 2001, 2001).

- Resumen: incluya un resumen de máximo 200 palabras, tanto en español o portugués como inglés.

- Palabras Clave: máximo seis palabras clave, preferiblemente complementarias al título del artículo, en español e inglés. 


\section{Agradecimientos}

Opcional. Párrafo sencillo y conciso entre el texto y la bibliografía. Evite títulos como Dr., Lic., TSU, etc.

\section{Fotografías, figuras, tablas y anexos}

Refiera las figuras (gráficas, diagramas, ilustraciones y fotografías) sin abreviación (p. e. Figura 3 ) al igual que las tablas (p. e. Tabla 1). Gráficos (p. e. CPUE anuales) y figuras (histogramas de tallas), preferiblemente en blanco y negro, con tipo y tamaño de letra uniforme. Deben ser nítidas y de buena calidad, evitando complejidades innecesarias (por ejemplo, tridimensionalidad en gráficos de barras); cuando sea posible use solo colores sólidos en lugar de tramas. Las letras, números o símbolos de las figuras deben ser de un tamaño adecuado de manera que sean claramente legibles una vez reducidas. Para el caso de las fotografías y figuras digitales es necesario que estas sean guardadas como formato tiff con una resolución de 300 dpi. Es oportuno que indique en qué parte del texto desea insertarla.

Lo mismo aplica para las tablas y anexos, los cuales deben ser simples en su estructura (marcos) y estar unificados. Presente las tablas en archivo aparte (Excel), identificadas con su respectivo número. Haga las llamadas a pie de página de tabla con letras ubicadas como superíndice. Evite tablas grandes sobrecargadas de información y líneas divisorias o presentadas en forma compleja. Es oportuno que indique en qué parte del texto desea insertar tablas y anexos.

\section{Bibliografía}

Contiene únicamente la lista de las referencias citadas en el texto. Ordénelas alfabéticamente por autores y cronológicamente para un mismo autor. Si hay varias referencias de un mismo autor(es) en el mismo año, añada las letras a, b, c, etc. No abrevie los nombres de las revistas. Presente las referencias en el formato anexo, incluyendo el uso de espacios, comas, puntos, mayúsculas, etc.

\section{ARTíCUlO EN REVISTAS}

Agosti, D., C. R. Brandao y S. Diniz. 1999. The new world species of the subfamily Leptanilloidinae (Hymenoptera: Formicidae). Systematic Entomology 24: 14-20.

LIBROS, TESIS E INFORMES TÉCNICOS

Libros: Gutiérrez, F. P. 2010. Los recursos hidrobiológicos y pesqueros en Colombia. Instituto de Investigación de Recursos Biológicos Alexander von Humboldt. Bogotá, D. C., 118 pp.

Tesis: Cipamocha, C. A. 2002. Caracterización de especies y evaluación trófica de la subienda de peces en el raudal Chorro de Córdoba, bajo río Caquetá, Amazonas, Colombia. Trabajo de grado. Universidad Nacional de Colombia, Facultad de Ciencias, Departamento de Biología. Bogotá D. C., 160 pp.

Informes técnicos: Andrade, G. I. 2010. Gestión del conocimiento para la gestión de la biodiversidad: bases conceptuales y propuesta programática para la reingeniería del Instituto Humboldt. Informe Técnico. Instituto de Investigación de Recursos Biológicos Alexander von Humboldt. Bogotá D. C., 80 pp.

Capitulo en libro o en informe: Fernández F., E. E. Palacio y W. P. MacKay. 1996. Introducción al estudio de las hormigas (Hymenoptera: Formicidae) de Colombia. Pp: 349-412. En: Amat, G. D., G. Andrade y F. Fernández (Eds.). Insectos de Colombia. Estudios Escogidos. Academia Colombiana de Ciencias Exactas, Físicas y Naturales \& Centro Editorial Javeriano, Bogotá.

Resumen en congreso, simposio, talleres: Señaris, J. C. 2001. Distribución geográfica y utilización del hábitat de las ranas de cristal (Anura; Centrolenidae) en Venezuela. En: Programa y Libro de Resúmenes del IV Congreso Venezolano de Ecología. Mérida, Venezuela, p. 124.

PÁginas Web

No serán incluidas en la bibliografía, sino que se señalarán claramente en el texto al momento de mencionarlas.

\section{Guidelines for authors}

(humboldt.org.co/es/bibliotecaypublicaciones/biota)

\section{Manuscript preparation}

Submitting a manuscript implies the explicit statement by the author(s) that the paper has not been published before nor accepted for publication in another journal or other means of scientific diffusion. Contributions are entire responsibility of the author and not the Alexander von Humboldt Institute for Research on Biological Resources, or the journal and their editors.

Papers can be written in Spanish, English or Portuguese and it is recommended not exceeding 40 pages (with paragraphs spaced at 1,5) including tables, figures and Annex. For special cases, the editor could consider publishing more extensive papers, monographs or symposium conclusions. New species descriptions for science, new geographic records and regional biodiversity lists are of particular interest for this journal.
Any word-processor program may be used for the text (Word is recommended). taxonomic list or any other type of table, should be prepared in spreadsheet aplication (Excel is recommended). To submit a manuscript must be accompanied by a cover letter which clearly indicate s:

1. Full names, mailing addresses and e-mail addresses of all authors. (Please note that email addresses are essential to direct communication).

2. The complete title of the article.

3. Names, sizes, and types of files provide.

4. A list of the names and addresses of at least three (3) reviewers who are qualified to evaluate the manuscript. 


\section{Evaluation}

Submitted manuscript will have a peer review evaluation. Resulting in any of the following: a) accepted (in this case we assume that no change, omission or addition to the article is required and it will be published as presented.); b) conditional acceptance (the article is accepted and recommended to be published but it needs to be corrected as indicated by the reviewer); and c) rejected (when the reviewer considers that the contents and/or form of the paper are not in accordance with requirements of publication standards of Biota Colombiana).

\section{Text}

- The manuscript specifications should be the following: standard letter size paper, with $2.5 \mathrm{~cm}$ margins on all sides, 1.5-spaced and left-aligned (including title and bibliography)

- All text pages (with the exception of the title page) should be numbered. Pages should be numbered in the lower right corner.

- Use Times New Roman or Arial font, size 12, for all texts. Use size 10 text in tables. Avoid the use of bold or underlining. 40 pages maximum, including tables, figures and annex. For tables use size 10 Times New Roman or Arial Font (the one used earlier).

- The manuscripts must be completed with the following order: title, abstract and key words, then in Spanish Título, Resumen y Palabras claves. Introduction, Materials and Methods, Results, Discussion, conclusions (optional), acknowledgements (optional) and bibliography. Following include a page with the Table, Figure and Annex list. Finally tables, figures and annex should be presented and clearly identified in separate tables.

- Scientific names of genera, species and subspecies should be written in italic. The same goes for Latin technical terms (i.e sensu, et al.). Avoid the use of underlining any word or title. Do not use footnotes.

- As for abbreviations and the metric system, use the standards of the International System of Units (SI) remembering that there should always be a space between the numeric value and the measure unit (e.g., $16 \mathrm{~km}, 23^{\circ} \mathrm{C}$ ). For relative measures such as $\mathrm{m} / \mathrm{sec}$, use $\mathrm{m} \cdot \mathrm{sec}^{-1}$.

- Write out numbers between one to ten in letters except when it precedes a measure unit (e.g., $9 \mathrm{~cm}$ ) or if it is used as a marker (e.g., lot 9, sample 7).

- Do not use a point to seperate thousands, millions, etc. Use a comma to separate the whole part of the decimal (e.g., 3,1416). Numerate the hours of the from 0:00 to 24:00. Express years with all numbers and without marking thousands (e.g., 19961998). In Spanish, the names of the months and days (enero, julio, sábado, lunes) are always written with the first letter as a lower case, but it is not this way in English.

- The cardinal points (north, south, east, and west) should always be written in lower case, with the excpetino of abbreviations $\mathrm{N}, \mathrm{S}, \mathrm{E}, \mathrm{O}$ (in English NW), etc. The correct indication of geographic coordinates is as follows: $02^{\circ} 37^{\prime} 53^{\prime \prime} \mathrm{N}-56^{\circ} 28^{\prime} 53^{\prime \prime} \mathrm{O}$. The geographic altitude should be cited as follows: $1180 \mathrm{~m}$ a.s.l.

- Abbreviations are explained only the first time they are used.
- When quoting references in the text mentioned author's last names when they are one or two, and et al. after the last name of the first author when there are three or more. If you mention many references, they should be in chronological order and separated by commas (e.g., Rojas 1978, Bailey et al. 1983, Sephton 2001, 2001).

- Abstract: include an abstract of 200 words maximum, in Spanish, Portuguese or English.

- KeY WORDS: six key words maximum, complementary to the title.

\section{Pictures, Figures, Tables and Annex}

- Figures (graphics, diagrams, illustrations and photographs) without abbreviation (e.g. Figure 3) the same as tables (e.g., Table 1). Graphics and figures should be in black and white, with uniform font type and size. They should be sharp and of good quality, avoiding unnecessary complexities (e.g., three dimensions graphics). When possible use solid color instead of other schemes. The words, numbers or symbols of figures should be of an adequate size so they are readable once reduced. Digital figures must be sent at $300 \mathrm{dpi}$ and in .tiff format. Please indicate in which part of the text you would like to include it.

- The same applies to tables and annexes, which should be simple in structure (frames) and be unified. Present tables in a separate file (Excel), identified with their respective number. Make calls to table footnotes with superscript letters above. Avoid large tables of information overload and fault lines or presented in a complex way. It is appropriate to indicate where in the text to insert tables and annexes.

\section{Bibliography}

References in bibliography contains only the list of references cited in the text. Sort them alphabetically by authors and chronologically by the same author. If there are several references by the same author(s) in the same year, add letters a, b, c, etc. Do not abbreviate journal names. Present references in the attached format, including the use of spaces, commas, periodss, capital letters, etc.

JOURNAL ARTICLE

Agosti, D., C. R. Brandao y S. Diniz. 1999. The new world species of the subfamily Leptanilloidinae (Hymenoptera: Formicidae). Systematic Entomology 24: 14-20.

BOOK, THESIS, TECHNICAL REVIEWS

Book: Gutiérrez, F. P. 2010. Los recursos hidrobiológicos y pesqueros en Colombia. Instituto de Investigación de Recursos Biológicos Alexander von Humboldt. Bogotá, D. C. 118 pp.

Thesis: Cipamocha, C. A. 2002. Caracterización de especies y evaluación trófica de la subienda de peces en el raudal Chorro de Córdoba, bajo río Caquetá, Amazonas, Colombia. Trabajo de grado. Universidad Nacional de Colombia, Facultad de Ciencias, Departamento de Biología. Bogotá D. C. 160 pp.

Technical reviews: Andrade, G. I. 2010. Gestión del conocimiento para la gestión de la biodiversidad: bases conceptuales y propuesta programática para la reingeniería del Instituto Humboldt. Informe 
Técnico. Instituto de Investigación de Recursos Biológicos Alexander von Humboldt. Bogotá D. C. 80 pp.

Book chapter or in review: Fernández F., E. E. Palacio y W. P. MacKay. 1996. Introducción al estudio de las hormigas (Hymenoptera: Formicidae) de Colombia. Pp:349-412. En: Amat, G. D., G. Andrade y F. Fernández (Eds.). Insectos de Colombia. Estudios Escogidos. Academia Colombiana de Ciencias Exactas, Físicas y Naturales \& Centro Editorial Javeriano, Bogotá.
Symposium abstract: Señaris, J. C. 2001. Distribución geográfica y utilización del hábitat de las ranas de cristal (Anura; Centrolenidae) en Venezuela. En: Programa y Libro de Resúmenes del IV Congreso Venezolano de Ecología. Mérida, Venezuela, p. 124.

WeB PAGES

Not be included in the literature, but clearly identified in the text at the time of mention.

\section{Guía para autores - Artículos de Datos}

www.humboldt.org.co/es/bibliotecaypublicaciones/biota- biotacol@humboldt.org.co

www.sibcolombia.net - sib+iac@humboldt.org.co

El objetivo de esta guía es establecer y explicar los pasos necesarios para la elaboración de un manuscrito con el potencial de convertirse en artículo de datos para ser publicado en la revista Biota Colombiana. En esta guía se incluyen aspectos relacionados con la preparación de datos y el manuscrito.

\section{¿Qué es un artículo de datos?}

Un artículo de datos o Data Paper es un tipo de publicación académica que ha surgido como mecanismo para incentivar la publicación de datos sobre biodiversidad, a la vez que es un medio para generar reconocimiento académico y profesional adecuado a todas las personas que intervienen de una manera u otra en la gestión de información sobre biodiversidad.

Los artículos de datos contienen las secciones básicas de un artículo científico tradicional. Sin embargo, estas se estructuran de acuerdo a un estándar internacional para metadatos (información que le da contexto a los datos) conocido como el GBIF Metadata Profile (GMP) ${ }^{1}$. La estructuración del manuscrito con base en este estándar se da, en primer lugar, para facilitar que la comunidad de autores que publican conjuntos de datos a nivel global, con presencia en redes como la Global Biodiversity Information Facility (GBIF) y otras redes relacionadas, puedan publicar fácilmente artículos de datos obteniendo el reconocimiento adecuado a su labor. En segundo lugar, para estimular que los autores de este tipo de conjuntos de datos que aún no han publicado en estas redes de información global, tengan los estímulos necesarios para hacerlo.

Un artículo de datos debe describir de la mejor manera posible el quién, qué, dónde, cuándo, por qué y cómo de la toma y almacenamiento de los datos, sin llegar a convertirse en el medio para realizar un análisis exhaustivo de los mismos, como sucede en otro tipo de publicaciones académicas. Para profundizar en este modelo de publicación se recomienda consultar a Chavan y Penev $(2011)^{2}$.

\begin{abstract}
¿Qué manuscritos pueden llegar a ser artículos de datos?
Manuscritos que describan conjuntos de datos primarios y originales que contengan registros biológicos (captura de datos de la presencia de un(os) organismo(s) en un lugar y tiempo determinados); información asociada a ejemplares de colecciones biológicas; listados temáticos o geográficos de especies; datos genómicos y todos aquellos datos que sean susceptibles de ser estructurados con el estándar Darwin Core ${ }^{3}$ (DwC). Este estándar es utilizado dentro de la comunidad de autores que publican conjuntos de datos sobre biodiversidad para estructurar los datos y de esta manera poder consolidarlos e integrarlos desde diferentes fuentes a nivel global. No se recomienda someter manuscritos que describan conjuntos de datos secundarios, como por ejemplo compilaciones de registros biológicos desde fuentes secundarias (p.e. literatura o compilaciones de registros ya publicados en redes como GBIF o IABIN).
\end{abstract}

\section{Preparación de los datos}

Como se mencionó anteriormente los datos sometidos dentro de este proceso deben ser estructurados en el estándar DwC. Para facilitar su estructuración, el Sistema de Información sobre Biodiversidad de Colombia (SiB Colombia), ha creado dos plantillas en Excel, una para registros biológicos y otra para listas de especies. Lea y siga detenidamente las instrucciones de las plantillas para la estructuración de los datos a publicar. Para cualquier duda sobre el proceso de estructuración de estos datos por favor contactar al equipo coordinador del SiB Colombia (ECSiB) en sib+iac@humboldt.org.co.

\footnotetext{
${ }^{1}$ Wieczorek, J. 2011. Perfil de Metadatos de GBIF: una guía de referencia rápida. En: Wieczorek, J. The GBIF Integrated Publishing Toolkit User Manual, version 2.0. Traducido y adaptado del inglés por D. Escobar. Sistema de Información sobre Biodiversidad de Colombia, Bogotá D.C., Colombia, 23p. Disponible en http://www.sibcolombia.net/repositorio-de-documentos.

${ }^{2}$ Chavan, V. y L. Penev. 2011. The data paper: The mechanism to incentivize data publishing in biodiversity science. BMC Bioinformatics 12 (Suppl 15): $\mathrm{S} 2$.

${ }^{3}$ TDWG. 2011. Darwin Core: una guía de referencia rápida. (Versión original producida por TDWG, traducida al idioma español por Escobar, D.; versión 2.0). Bogotá: SiB Colombia, 33 pp. Disponible en http://www.sibcolombia.net/repositorio-de-documentos
} 


\section{Preparación del manuscrito}

Para facilitar la creación y estructuración del manuscrito en el estándar GMP, se cuenta con la ayuda de un editor electrónico (http://ipt.sibcolombia.net/biota) que guiará al autor en dicho proceso y que finalmente generará una primera versión del manuscrito. Se recomienda el uso del manual GMP, como una guía de la información a incluir en cada sección del manuscrito, junto con el anexo 1.

Pasos a seguir para la elaboración del manuscrito:

1 Solicitealcorreosib+iac@humboldt.org.coelaccesoaleditor electrónico. El EC-SiB le asignará un usuario y contraseña.

2. Ingreseconsuusuarioycontraseñaaleditorelectrónico,luego diríjase a la pestaña Gestión de recursos y cree un nuevo recurso asignando un nombre corto a su manuscrito usando el formato "AcrónimoDeLaInstitución_año_tipoDeConjuntoDeDatos", p.e. ABC_2010_avestinije y dar clic en el botón crear.

3. Enlavistageneraldeleditorseleccione "editar"enlapestaña Metadatos (por favor, no manipule ningún otro elemento), allí encontrará diferentes secciones (panel derecho) que lo guiarán en la creación de su manuscrito. Guarde los cambios al finalizar cada sección, de lo contrario perderá la información. Recuerde usar el manual GMP. A continuación se presentan algunas recomendaciones para la construcción del manuscrito. Las secciones se indican en MAYUSCULAS y los elementos de dichas secciones en negrilla.

- $\quad$ En PARTES ASOCIADAS incluya únicamente aquellas personas que no haya incluido en INFORMACIÓN BÁSICA.

- Los DATOS DEL PROYECTO y DATOS DE LA COLECCIÓN son opcionales según el tipo de datos. En caso de usar dichas secciones amplíe o complemente información ya suministrada, p. ej. no repita información de la descripción (COBERTURA GEOGRÁFICA) en la descripción del área de estudio (DATOS DEL PROYECTO).

- De igual manera, en los MÉTODOS DE MUESTREO, debe ampliar o complementar información, no repetirla. La información del área de estudio debe dar un contexto específico a la metodología de muestreo.

- Es indispensable documentar el control de calidad en MÉTODOS DE MUESTREO. Acá se debe describir que herramientas o protocolos se utilizaron para garantizar la calidad y coherencia de los datos estructurados con el estándar DwC.

- Para crear la referencia del recurso, en la sección REFERENCIAS, utilice uno de los dos formatos propuestos (Anexo 2). No llene el identificador de la referencia, este será suministrado posteriormente por el EC-SiB.

- Para incluir la bibliografía del manuscrito en referencias, ingrese cada una de las citas de manera individual, añadiendo una nueva referencia cada vez haciendo clic en la esquina inferior izquierda.

4. Rectifique que el formato de la información suministrada cumpla con los lineamientos de la revista (p. ej. abreviaturas, unidades, formato de números etc.) en la Guía general para autores de Biota Colombiana.

5. Una vez incluida y verificada toda la información en el editor electrónico notifique al EC-SiB al correo electrónico sib+iac@ humboldt.org.co, indicando que ha finalizado la edición del manuscrito. Adicionalmente adjunte la plantilla de Excel con los datos estructurados (elimine todas las columnas que no utilizó). El EC-SiB realizará correcciones y recomendaciones finales acerca de la estructuración de los datos y dará las instrucciones finales para que usted proceda a someter el artículo.

\section{Someter el manuscrito}

Una vez haya terminado la edición de su manuscrito y recibido las instrucciones por parte del EC-SIB, envíe una carta al correo electrónico biotacol@humboldt.org.co para someter su artículo, siguiendo las instrucciones en la Guía general para autores de Biota Colombiana.

Recuerde adjuntar:

- Plantilla de Excel con la última versión de los datos revisada por el EC-SiB.

- Documento de Word con las figuras y tablas seguidas de una lista las mismas.

Cuando finalice el proceso, sus datos se harán públicos y de libre acceso en los portales de datos del SiB Colombia y GBIF. Esto permitirá que sus datos estén disponibles para una audiencia nacional e internacional, manteniendo siempre el crédito para los autores e instituciones asociadas. 
Anexo 1. Estructura base de un artículo de datos y su correspondencia con el editor electrónico basado en el GMP.

\begin{tabular}{|c|c|}
\hline SECCIÓN/SUBSECCIÓN & CORRESPONDENCIA CON LOS ELEMENTOS DEL EDITOR ELECTRÓNICO \\
\hline Título & Derivado del elemento título. \\
\hline AutORES & Derivado de los elementos creador del recurso, proveedor de los metadatos y partes asociadas. \\
\hline AfILIACIONES & $\begin{array}{l}\text { Derivado de los elementos creador del recurso, proveedor de los metadatos y partes asociadas. } \\
\text { De estos elementos, la combinación de organización, dirección, código postal, ciudad, país y } \\
\text { correo electrónico, constituyen la afiliación. }\end{array}$ \\
\hline AUTOR DE CONTACTO & Derivado de los elementos creador del recurso y proveedor de los metadatos. \\
\hline CITACIÓN & Para uso de los editores. \\
\hline CitACión DELE RECURSO & Derivada del elemento referencia del recurso. \\
\hline RESUMEN & Derivado del elemento resumen. Máximo 200 palabras. \\
\hline Palabras Clave & Derivadas del elemento palabras clave. Máximo seis palabras. \\
\hline AbSTRACT & Derivado del elemento abstract. Máximo 200 palabras. \\
\hline KEY WORDS & Derivadas del elemento key words. Máximo seis palabras. \\
\hline INTRODUCCIÓN & $\begin{array}{l}\text { Derivado del elemento propósito (de las secciones Introducción y Antecedentes). Se sugiere un } \\
\text { breve texto para introducir las siguientes secciones. Por ejemplo, historia o contexto de la colección } \\
\text { biológica o proyecto en relación con los datos descritos, siempre y cuando no se repita información } \\
\text { en las subsecuentes secciones. }\end{array}$ \\
\hline Datos del proyecto & $\begin{array}{l}\text { Derivada de los elementos de la sección Datos del proyecto: título, nombre, apellido, rol, fuentes } \\
\text { de financiación, descripción del área de estudio y descripción del proyecto. }\end{array}$ \\
\hline Cobertura taxonómica & $\begin{array}{l}\text { Derivada de los elementos de la sección Cobertura taxonómica: descripción, nombre científico, } \\
\text { nombre común y categoría. }\end{array}$ \\
\hline Cobertura geográfica & $\begin{array}{l}\text { Derivada de los elementos de la sección Cobertura geográfica: descripción, latitud mínima, } \\
\text { latitud máxima, longitud mínima, longitud máxima. }\end{array}$ \\
\hline Cobertura temporal & Derivada de los elementos de la sección Cobertura temporal: tipo de cobertura temporal. \\
\hline Datos de la colección & $\begin{array}{l}\text { Derivada de los elementos de la sección Datos de la colección: nombre de la colección, } \\
\text { identificador de la colección, identificador de la colección parental, método de preservación } \\
\text { de los especímenes y unidades curatoriales. }\end{array}$ \\
\hline MATERIAL Y MÉTODOS & $\begin{array}{l}\text { Derivado de los elementos de la sección Métodos de muestreo: área de estudio, descripción del } \\
\text { muestreo, control de calidad, descripción de la metodología paso a paso. }\end{array}$ \\
\hline \multicolumn{2}{|r|}{ ( } \\
\hline Descripción del conjunto de datos & $\begin{array}{l}\text { Derivado de los elementos de las secciones Discusión y Agradecimientos, contiene información } \\
\text { del formato de los datos y metadatos: nivel de jerarquía, fecha de publicación y derechos de } \\
\text { propiedad intelectual. }\end{array}$ \\
\hline DisCUSIÓN & $\begin{array}{l}\text { Se deriva del elemento discusión. Un texto breve (máximo } 500 \text { palabras), que puede hacer } \\
\text { referencia a la importancia, relevancia, utilidad o uso que se le ha dado o dará a los datos en } \\
\text { publicaciones existentes o en posteriores proyectos. }\end{array}$ \\
\hline AgRADECIMIENTOS & Se deriva del elemento agradecimientos. \\
\hline BIBLIOGRAFÍA & Derivado del elemento bibliografía. \\
\hline
\end{tabular}


Guía para autores - Artículos de Datos / Guidelines for authors - Data Papers

Anexo 2. Formatos para llenar el elemento referencia del recurso.

La referencia del recurso es aquella que acompañará los datos descritos por el artículo, públicos a través de las redes SiB Colombia y GBIF. Tenga en cuenta que esta referencia puede diferir de la del artículo. Para mayor información sobre este elemento contacte al EC-SiB. Aquí se sugieren dos formatos, sin embargo puede consultar otros formatos establecidos por $\mathrm{GBIF}^{4}$.

\begin{tabular}{ll}
\hline TIPO DE RECURSO & \multicolumn{1}{c}{ PLANTILLA } \\
\hline El conjunto de datos que & $<$ Institución publicadora/Grupo de investigación $>$ \\
el manuscrito describe es & $<($ Año $)>,<$ Título del recurso/Artículo $>$. $<$ Número \\
resultado de un proyecto & total de registros $>,<$ aportados por: $><$ parte \\
de carácter institucional & asociada $1($ rol $)$, parte asociada $2($ rol $)(\ldots)>$. $<$ En \\
o colectivo con múltiples & línea, $><$ url del recurso $>$. $<$ Publicado el DD/MM/ \\
participantes. & AAAA $>$
\end{tabular}

El conjunto de datos que el manuscrito describe es resultado de una iniciativa personal o de un grupo de investigación definido.
$<$ Parte asociada 1, parte asociada $2(\ldots)>$ $<$ (Año) $>,<$ Título del recurso/Artículo $>$, $<$ Número total de registros $>$, $<$ en línea, $><$ url del recurso $>$. $<$ Publicado el DD/MM/AAAA $>$

\section{EJEMPLO}

Centro Nacional de Biodiversidad (2013). Vertebrados de la cuenca de la Orinoquia. 1500 registros, aportados por Pérez, S. (Investigador principal, proveedor de contenidos, proveedor de metadatos), M. Sánchez (Procesador), D. Valencia (Custodio, proveedor de metadatos), R. Rodríguez (Procesador), S. Sarmiento (Publicador), V. B. Martínez (Publicador, editor). En línea, http://ipt.sibcolombia.net/biota/resource.do? $\mathrm{r}=$ verte_orin, publicado el 01/09/2013.

Valencia, D., R. Rodríguez y V. B. Martínez (2013). Vertebrados de la cuenca del Orinoco. 1500 registros, en línea, http://ipt.sibcolombia.net/biota/ resource.do?r=verte_orin. Publicado el 01/09/2001.

\section{Guidelines for authors - Data Papers}

www.humboldt.org.co/es/bibliotecaypublicaciones/biota-biotacol@humboldt.org.co|

www.sibcolombia.net - sib+iac@humboldt.org.co

The purpose of this guide is to establish and explain the necessary steps to prepare a manuscript with the potential to become a publishable data paper in Biota Colombiana. This guide includes aspects related to the preparation of both data and the manuscript.

\section{What is a Data Paper?}

A data paper is a scholarly publication that has emerged as a mechanism to encourage the publication of biodiversity data as well as an approach to generate appropriate academic and professional recognition to all those involved in in the management of biodiversity information.

A data paper contains the basic sections of a traditional scientific paper. However, these are structured according to an international standard for metadata (information that gives context to the data) known as the GBIF Metadata Profile (GMP) ${ }^{5}$. The structuring of the manuscript based on this standard enables the community of authors publishing datasets globally, with presence in networks such as the Global Biodiversity Information Facility (GBIF) and other related networks, to publish data easily while getting proper recognition for their work and to encourage the authors of this type of data sets that have not yet published in these global information networks to have the necessary incentives to do so.

A data paper should describe in the best possible way the Whom, What, Where, When, Why and How of documenting and recording of data, without becoming the instrument to make a detailed analysis of the data, as happens in other academic publications. To deepen this publishing model, it is recommended to consult Chavan \& Penev $(2011)^{6}$.

\footnotetext{
${ }^{4}$ GBIF (2012). Recommended practices for citation of the data published through the GBIF Network. Version 1.0 (Authored by Vishwas Chavan), Copenhagen: Global Biodiversity Information Facility. Pp.12, ISBN: 87-92020-36-4. Accessible at http://links.gbif.org/gbif_best_practice_data_citation_ en_v1

${ }^{5}$ GBIF (2011). GBIF Metadata Profile, Reference Guide, Feb 2011, (contributed by O Tuama, E., Braak, K., Copenhagen: Global Biodiversity Information Facility, 19 pp. Accesible at http://links.gbif.org/gbif_metadata_profile_how-to_en_v1.

${ }^{6}$ Chavan, V. y L. Penev. 2011. The data paper: The mechanism to incentivize data publishing in biodiversity science. BMC Bioinformatics 12 (Suppl 15): S2.
} 


\section{Which manuscripts are suitable for publication as data paper?}

Manuscripts that describe datasets containing original primary biological records (data of occurrences in a particular place and time); information associated with specimens of biological collections, thematic or regional inventories of species, genomic data and all data likely to be structured with the standard Darwin CoreDarwin Core $(\mathrm{DwC})$. This standard is used in the community of authors publishing biodiversity datasets to structure the data and thus to consolidate and integrate from different sources globally. It is not recommended to submit manuscripts describing secondary datasets, such as biological records compilations from secondary sources (e.g. literature or compilations of records already published in networks such as GBIF or IABIN).

\section{Dataset preparation}

As mentioned above data submitted in this process should be structured based on DwC standard. For ease of structuring, the Biodiversity Information System of Colombia (SiB Colombia), created two templates in Excel; one for occurrences and other for species checklist. Carefully read and follow the template instructions for structuring and publishing data. For any questions about the structure process of data please contact the Coordinator Team of SiB Colombia (EC-SiB) at sib+iac@humboldt.org.co

\section{Manuscript preparation}

To assist the creation and structuring of the manuscript in the GMP standard, an electronic writing tool is available (http://ipt. sibcolombia.net/biota) to guide the author in the process and ultimately generate a first version of the manuscript. The use of GMP manual as an information guide to include in each section of the manuscript, as well as the annex 1 is recommended.

Steps required for the manuscript preparation:

1 Request access to the electronic writing tool at sib+iac@ humboldt.org.co. The EC-SiB will assign a username and password.

2. Login to the electronic writing tool, then go to the tab Manage Resources and create a new resource by assigning a short name for your manuscript and clicking on the Create button. Use the format: "InstitutionAcronym_Year_DatasetFeature", e.g. NMNH_2010_rainforestbirds.

3. In the overview of the writing tool click on edit in Metadata section (please, do not use any other section), once there you will find different sections (right panel) that will guide you creating your manuscript. Save the changes at the end of each section, otherwise you will lose the information. Remember to use the GMP manual. Here are some recommendations for editing the metadata, sections are indicated in CAPS and the elements of these sections in bold.
- In ASSOCIATED PARTIES include only those who are not listed in BASIC INFORMATION.

- PROJECT DATA and COLLECTION DATA are optional depending on the data type. When using these sections extend or complement information already provided, i.e. do not repeat the same information describing the description (GEOGRAPHIC COVERAGE) in the study area description (PROJECT DATA).

- Likewise, in SAMPLING METHODS, you must expand or complete the information, not repeat it. The information in study extent should give a specific context of the sampling methodology.

- It is essential to document the quality control in SAMPLING METHODS. Here you should describe what tools or protocols were used to ensure the quality and consistency of data structured with DwC standard.

- To create the resource citation in the CITATIONS section, follow one of the two formats proposed (Annex 2). Do not fill out the citation identifier, this will be provided later by the EC-SiB.

- To include the manuscript bibliography in citations, enter each of the citations individually, adding a new citation each time by clicking in the bottom left.

4. Check that the format of the information provided meets the guidelines of the journal (e.g. abbreviations, units, number formatting, etc.) in the Biota Colombiana Guidelines for Authors.

5. Once included and verified all information in the writing tool, notify to EC-SiB at sib+iac@humboldt.org.co, indicating that you have finished editing the manuscript. Additionally attach the Excel template with structured data (remove all columns that were not used). The EC-SiB will perform corrections and final recommendations about the structure of the data and give you the final instructions to submit the paper.

\section{Submit the manuscript}

Once you have finished editing your manuscript and getting the instructions from EC-SIB, send a letter submitting your article to email biotacol@humboldt.org.co, following the instructions of Biota Colombiana Guidelines for Authors.

Remember to attach:

- Excel template with the latest version of the data reviewed by the EC-SiB.

- Word document with figures and tables followed by a list of them.

At the end of the process, your information will be public and freely accessible in the data portal of SiB Colombia and GBIF. This will allow your data to be available for national and international audience, while maintaining credit to the authors and partner institutions.

\footnotetext{
${ }^{7}$ Biodiversity Information Standards - TDWG. Accesible at http://rs.tdwg.org/dwc/terms/
} 
Annex 1. Basic structure of a data paper and its mapping to the writing tool elements based on GM.

\section{SECTION/SUB-SECTION HEADING \\ MAPPING WITH WRITING TOOL ELEMENTS}

TitLE Derived from the title element.

AUTHORS Derived from the resource creator, metadata provider, and associated parties elements.

AfFILIATIONS Derived from the resource creator, metadata provider and associated parties elements. From these elements combinations of organization, address, postal code, city, country and email constitute the affiliation.

CORRESPONDING AUTHOR Derived from the resource contact, metadata provider elements.

Citation For editors use.

RESOURCE CITATION Derived from the resource citation element.

RESUMEN Derived from the resumen element. 200 words max.

Palabras Clave Derived from the palabras clave element. 6 words max.

ABSTRACT Derived from the abstract element. 200 words max.

KEY WORDS Derived from the key words element. 6 words max.

INTRODUCTION Derived from the purpose (Introduction and Background section). A short text to introduce the following sections is suggested. For example, history or context of the biological collection or project related with the data described, only if that information is not present in subsequent sections.

Project data Derived from elements title, personnel first name, personnel last name, role, funding, study area description, and design description.

Taxonomic Coverage Derived from the taxonomic coverage elements: description, scientific name, common name and rank.

Geographic Coverage Derived from the geographic coverage elements: description, west, east, south, north.

Temporal Coverage Derived from the temporal coverage elements: temporal coverage type.

Collection data

MATERIALS AND METHODS

Derived from the collection data elements: collection name, collection identifier, parent collection identifier, specimen preservation method and curatorial units.

Derived from the sampling methods elements: study extent, sampling description, quality control and step description.

REsUltados

Descripción del conjunto de datos

Derived from the discussion and acknowledgments, contains information about the format of the data and metadata: hierarchy level, date published and ip rights.

Discussion Derived from the discussion element. A short text (max 500 words), which can refer to the importance, relevance, usefulness or use that has been given or will give the data in the published literature or in subsequent projects.

ACKNOWLEDGMENTS Derived from the acknowledgments element.

BiBLIOGRAPHY Derived from the citations element. 
Annex 2. Citation style quick guide for "resource reference" section.

The Resource Reference is the one that refer to the dataset described by the paper, publicly available through SiB Colombia and GBIF networks. Note that this reference may differ from the one of the paper. For more information about this element contact EC-SiB.

Here two formats are suggested; however you can consult other formats established by GBIF.

\section{TYPE OF RESOURCE}

The paper is the result of a collective or institutional project with multiple participants.

\section{TEMPLATE}

$<$ Institution/Research Group $>$. $<$ Year $>$, <Title of the Resource/Paper $>$. $<$ Number of total records $>$, <provided by :> <associated party 1 (role), associated party 2 (role), (..)>. < Online, $>$ $<$ resource URL $>$, $<$ published on $>$. $<$ Published on DD/MM/AAAA $>$.

$<$ associated party 1 , associated party $2,(\ldots)>$. $<$ Year $>$, $<$ Title of the Resource/Paper $>$, $<$ Number of total records $>,<$ Online, $><$ resource URL $>$. $<$ Published on DD/MM/AAAA $>$.

\section{EXAMPLE}

National Biodiversity (2013). Vertebrates in Orinoco, 1500 records, provided by: Perez, S. (Principal investigator, content provider), M. Sanchez (Processor), D. Valencia (Custodian Steward, metadata provider), R. Rodriguez (Processor), S. Sarmiento (Publisher), VB Martinez (Publisher, Editor). Online, http://ipt.sibcolombia.net/ biota/resource.do?r= verte_orin, published on 01/09/2013.

Valencia, D., R. Rodríguez and V. B. Martínez. (2013). Vertebrate Orinoco Basin, 1500 records, Online, http://ipt.sibcolombia.net/biota/resource. do? $\mathrm{r}=$ verte_orin, published on 01/09/2001

\footnotetext{
${ }^{8}$ GBIF (2012). Recommended practices for citation of the data published through the GBIF Network. Version 1.0 (Authored by Vishwas Chavan), Copenhagen: Global Biodiversity Information Facility. Pp.12, ISBN: 87-92020-36-4. Accessible at http://links.gbif.org/gbif_best_practice_data_citation_ en_v1
} 


\section{TABLA DE CONTENIDO / TABLE OF CONTENTS}

Presentación. Brigitte L. G. Baptiste, Carlos A. Lasso, Wilson Ramírez y Mauricio Aguilar-Garavito.

Introducción. Alianzas para la restauración de ecosistemas. I Simposio Regional de Restauración Ecológica Nodo REDCRE Suroccidente. Introduction. Alliance for restoration of ecosystems I Regional Symposium on Ecological Restoration REDCRE Node Southwest. Mauricio Aguilar-Garavito, Diana Catalina Rondón Camacho y William Vargas

Áreas naturales de bosque seco tropical en el Valle del Cauca, Colombia: una oportunidad para la restauración. Natural areas of tropical dry forest in Valle del Cauca, Colombia: an opportunity for restoration. Diana P. Alvarado-Solano y Joel T. Otero-Ospina

Lectura de un paisaje estratificado: propuesta de restauración basada en el ordenamiento multi-escala de las cañadas en la mesa de Xéridas, Santander, Colombia. Reading a stratified landscape: restoration proposal based on the multiscale ordination of the canebrakes at Mesa de Xéridas, Santander, Colombia. Germán Camargo-Ponce de León y Laura G. Agudelo-Álvarez

Experiencia piloto de nucleación con especies nativas para restaurar una zona degradada por ganadería en el norte de Antioquia, Colombia. A pilot nucleation experiment with native species to restore an area degraded by livestock in the north of Antioquia, Colombia. Mónica Díaz-Páez y Jaime Polanía .....

Zonificación de alternativas de conectividad ecológica, restauración y conservación en las microcuencas Curubital, Mugroso, Chisacá y Regadera, cuenca del río Tunjuelo (Distrito Capital de Bogotá), Colombia. Zonification of alternatives for ecological connectivity, restoration and conservation of the Curubital, Mugroso, Chisacá and Regadera microdrainages of the Tunjuelo River (Capital District of Bogotá), Colombia. Paola Isaacs Cubides, Ledy Trujillo y Vilma Jaimes

Banco de semillas de retamo espinoso Ulex europaeus L. en bordes del matorral invasor en un ecosistema zonal de bosque altoandino, Colombia. Seed bank of the spiny reed, Ulex europaeus L., along edges of thickets of this invasive species in a zonal ecosystem of High Andes forest, Colombia. Korina Ocampo-Zuleta y Jairo Solorza Bejarano .....

Forestación de bosques en sabanas de la altillanura colombiana: relevancia de las condiciones ambientales para el establecimiento de plántulas. Afforestation of savanna forests of the Colombian altillanura: relevance of the environmental conditions for the establishment of seedlings. Pablo R. Stevenson, Mónica A. Ramírez, Luisa F. Casas y Francisco Henao-Díaz 